\title{
Movilidad urbana post pandemia: fuerzas en pugna con sentidos de sustentabilidad contrapuestos
}

Andrés Borthagaray

Fundación Furban; Instituto para la ciudad en movimiento (América Latina); Facultad de Arquitectura, Diseño y Urbanismo, Universidad de Buenos Aires, Argentina.

ORCID: https://orcid.org/0000-0003-0781-5539

\author{
Andrea Gutiérrez \\ Universidad de Buenos Aires, Facultad de Filosofía y Letras, Instituto de Geografía, Programa \\ Transporte y Territorio; CONICET, Argentina. \\ ORCID: https://orcid.org/oooo-ooo2-8124-1679
}

Recibido: 30 de mayo de 2021. Aceptado 29 de junio de 2021.

\section{Resumen}

Este ensayo aporta una reflexión orientada a sintetizar, explorar e interpretar algunas experiencias y tendencias sobre esta encrucijada significativa planteada por la pandemia y sobre la consecuente posibilidad de cambios a futuro. Las restricciones a la movilidad han generado consecuencias en actividades esenciales, como el trabajo, la educación y el propio acceso a la salud. Si bien han existido medidas y alternativas de mitigación, estas se han dado y distribuido de modo desigual. Como parte de los escenarios posteriores a la emergencia, se proyecta una nueva organización de los tiempos y los espacios de la movilidad, y por su intermedio, del territorio y la distribución de oportunidades. Pero esta proyección permite anticipar tendencias encontradas que podrían incluso agudizar la anterior polarización. La movilidad activa, las mejoras de un transporte público adaptado a una demanda en evolución y la organización social de una movilidad sustentable se contraponen con una huida de la ciudad, un refugio en soluciones individuales para quienes pueden afrontarlas y una rigidez en la organización de servicios desde la oferta, llevados por las inercias territoriales, sociales e institucionales que generan una fuerte tracción ante los cambios de curso. 


\title{
Post-pandemic urban mobility: forces in tension with conflicting senses of sustainability
}

\begin{abstract}
This essay provides a reflection aimed at synthesizing, exploring, and interpreting some experiences and trends on this significant crossroads posed by the pandemic and the consequent possibility of future changes. Restrictions on mobility have had consequences for essential activities, such as work, education, and access to health itself. Although there have been mitigation measures and alternatives, they have been unevenly distributed and distributed. Post-emergency scenarios project a new organization of time and space of mobility, which implies a new configuration of the territory and distribution of opportunities. But this projection makes it possible to anticipate trends found. Active mobility, improvements in public transport adapted to an evolving demand, and the social organization of sustainable mobility contrast with a flight from the city, a refuge in individual solutions for those who can face them, and a rigidity in the organization of services from the supply, driven by territorial, social and institutional inertia that generate strong traction in the face of current changes.
\end{abstract}

Keywords: Mobility. Urbanism. Pandemic. Sustainability. Government. Society.

Palavras chave: Mobilidade. Urbanismo. Pandemia. Sustentabilidade. Governo. Sociedade.

\section{Movilidad urbana en la pandemia}

La pandemia tiene al desplazamiento territorial en su propio corazón. La movilidad de las personas fue parte de sus causas, y en consecuencia es parte de sus principales efectos -como medida preventiva y a partir de las acciones adoptadas- sobre toda la actividad humana en el territorio y sobre las personas. Por ende, hacer un barrido de las experiencias aparejadas por la pandemia en cuanto a movilidad y ciudad es algo tan amplio -en cobertura geográfica y en dimensiones de análisis- como imposible de realizar con exhaustividad. Pero esto mismo, tampoco es posible hacerlo con rigor analítico sin mediar un desarrollo metodológico que le aporte factibilidad, y que en sí mismo resulte un aporte conceptual.

Este ensayo carece de ambas pretensiones. Su objetivo es hilvanar una selección sintética de experiencias fundamentadas, para iluminar encrucijadas cuya exploración se considera significativa. Una licencia que la pandemia amerita, tratándose de un proceso en curso e inédito en la historia. Esto último no refiere tanto a su alcance mundial sino al hecho de ser una experiencia global vivida en tiempo simultáneo. Como tal, asimila un acontecimiento común -la pandemia- y lo traduce en innúmeras formas conforme a las especificidades locales (Gutiérrez, 2020).

Así, ante las sucesivamente prorrogadas expectativas de un levantamiento progresivo del lock down generado por la crisis del COVID 19, aún perduran las preguntas sobre cómo movernos manteniendo condiciones sanitarias de seguridad (ahora y a futuro). Movernos implica trabajar, estudiar, comprar, divertirnos, encontrarnos con familiares y amigos, además de cuidar la salud física y mental, entre otros aspectos que hacen a la vida misma.

Las restricciones al desplazamiento territorial se han traducido en pérdidas de empleo, caídas de la producción, rupturas de cadenas de pago y pérdida de ritmos escolares que, entre otras consecuencias, obligan a pensar alternativas. Es cierto que en parte se han atenuado por medidas sociales, de mitigación económica, por la conectividad 
y el desarrollo de actividades a distancia. Pero el acceso a esta última posibilidad se ha visto marcado por niveles de ingreso y tipo de actividad, lo que ha agravado las desigualdades existentes en el punto de partida.

Como efecto colateral, algunas estimaciones preliminares y tangibles, aunque efímeras, indican un descenso drástico en la cantidad de siniestros y muertes por accidentes de tránsito - ya consideradas epidemia por la Organización Mundial de la Salud (World Health Organization, 2009)-, y en la emisión de gases de efecto invernadero y micro partículas (causantes de otras enfermedades respiratorias). La divulgación de un estudio sobre China menciona un saldo neto sustantivamente menor de muertes, aun en el punto más alto de la pandemia en ese país (McMahon, 2020).

La cuestión es si lo vivido permitirá un nuevo punto de arranque que modifique (o revierta) las consecuencias más negativas del modelo o patrón de movilidad urbana previo a la crisis del COVID y, al mismo tiempo, si este nuevo punto de arranque podrá ofrecer desde las ciudades oportunidades más equitativas y menos vulnerables ante crisis estructurales, como la del calentamiento global (IPCC, 2018). En esta línea de reflexión, merece una consideración especial el impacto en el territorio y en los motivos de los viajes, en la organización de los hogares, de las actividades y de las áreas de mayor convergencia de ofertas de transporte público, como los grandes centros de las regiones metropolitanas.

La agenda internacional parecía haber arribado a un consenso -al menos en cuanto a los principios- sobre cómo tenía que orientarse la movilidad del futuro: menos dependencia del automóvil particular y de los combustibles fósiles, más y mejor transporte público y seguridad e incentivos para los desplazamientos a pie y en bicicleta; una revalorización de los centros urbanos y zonas de una densidad relativamente alta con actividades mixtas, más cargas por ferrocarril y por vías fluviales y marítimas y menos por ruta, por ejemplo (Naciones Unidas, 2017; International Energy Agency, 2021; International Transportation Forum, 2020), o la iniciativa Decarbonizing transport de ITF en general y en América Latina en particular (International Transportation Forum, 2021).

Paradójicamente, en este año se manifiesta también una tendencia incipiente en otra dirección: refugiarse, para quienes es posible, en el automóvil y la periferia de las ciudades. Una muestra puntual de esta situación se refleja, por lo menos en el corto plazo, en cambios en la demanda inmobiliaria por zona, ilustrada por análisis de valores en distintos contextos (Anzillotti, 2021; Kamin, 2021).

Frente a algunos discursos que asocian los niveles de contagio con ciertos niveles de densidad, cabría revisar en profundidad las estadísticas de algunas de las ciudades asiáticas más densas y mejor servidas por transporte público, que han podido llevar la crisis sin tener que recurrir a cuarentenas prolongadas y mucho antes de que se desarrollaran las distintas posibilidades de vacunación (Angel, Blei, Lamson-hall, y Salazar, 2020). Es cierto, con una cultura de prevención y trazabilidad muy particular. También es cierto, por otra parte, que las ciudades han salido fortalecidas de situaciones extremas, como por ejemplo de las pestes de cólera y fiebre amarilla, mediante el desarrollo de sistemas sanitarios innovadores (Deaton, 2015).

En ese sentido, y yendo específicamente a cómo nos movemos hoy, está claro que existe (y existirá) una tensión entre un transporte público que vaya teniendo una demanda creciente, y la necesidad de mantener las distancias sanitarias recomendadas. Frente a esa tensión, y frente a un reclamo de refugio individualista que puede funcionar solo para una fracción de la sociedad (y parcialmente), las medidas en curso han tendido a alinear objetivos por el lado de la oferta y por el lado de la demanda. 
Por el lado de la oferta, varias ciudades han buscado la forma de ampliar el espacio para los peatones, de modo tal de mantener las distancias recomendadas de -en lo posible1,5 metros. Más allá de los enunciados precedentes a favor de los peatones -movilidad entendida como la más eficiente y saludable-, las veredas (o aceras) han sido objeto de una pugna distributiva: quienes caminan deben competir en y por el espacio con carteles publicitarios, el estacionamiento de motos y bicicletas, los contenedores de residuos, y otros usos conflictivos que incluyen la eliminación de árboles para permitir la entrada a garages (Figura 1).

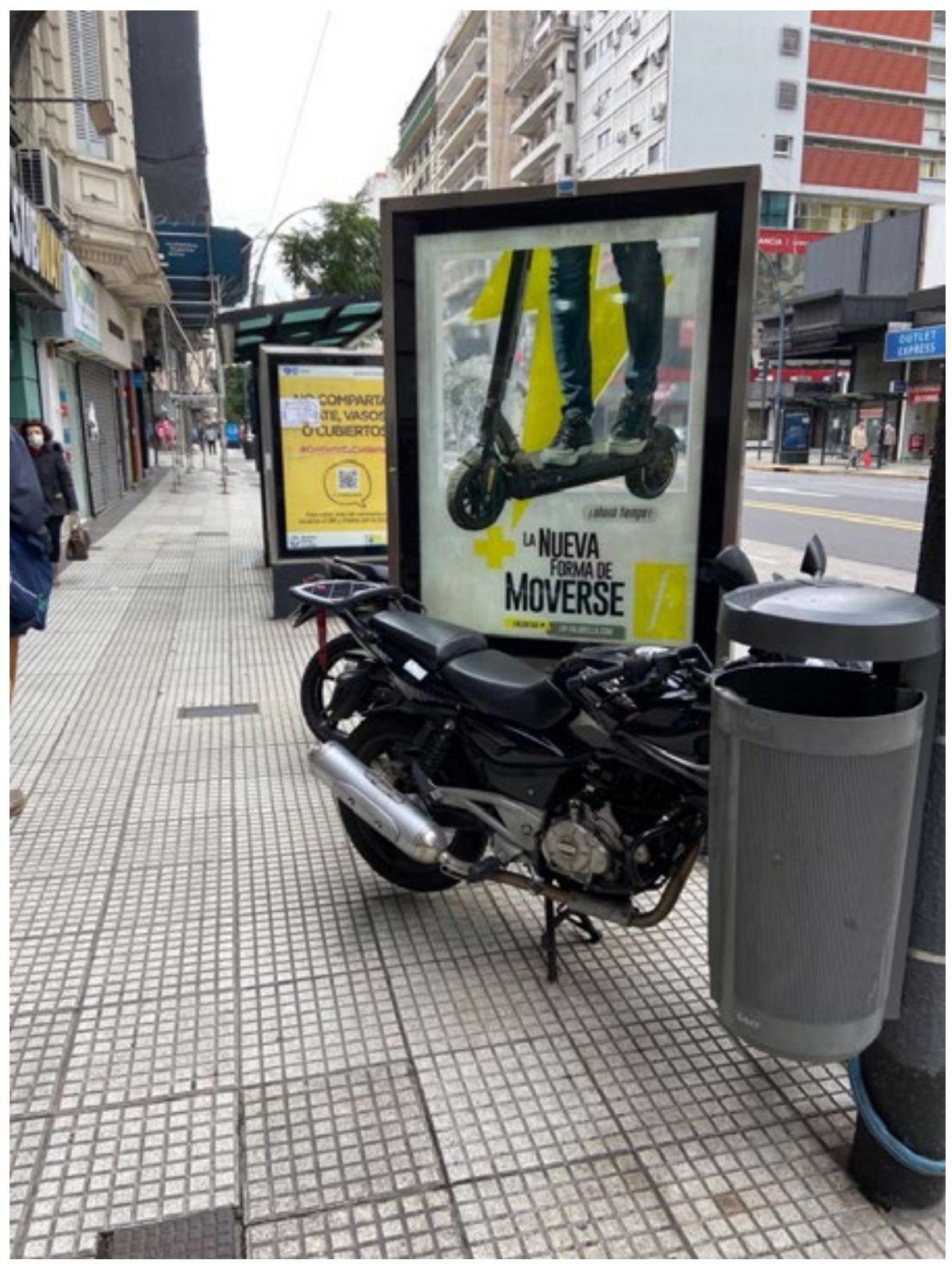

Figura 1. Puja distributiva en el espacio peatonal. Fuente: foto de los autores, 31/08/2020.

O bien, como ocurre en distintas partes del conurbano bonaerense -y otros conurbanos de Argentina y de América Latina- un equipamiento básico como es la vereda seca en días de lluvia se hace escaso, tanto así que incluso afecta en muchos barrios el acceso a las paradas del transporte público (Gutiérrez, 2012, Fernández, S., Scholl, L.y IADB, 2020) (Figura 2). 


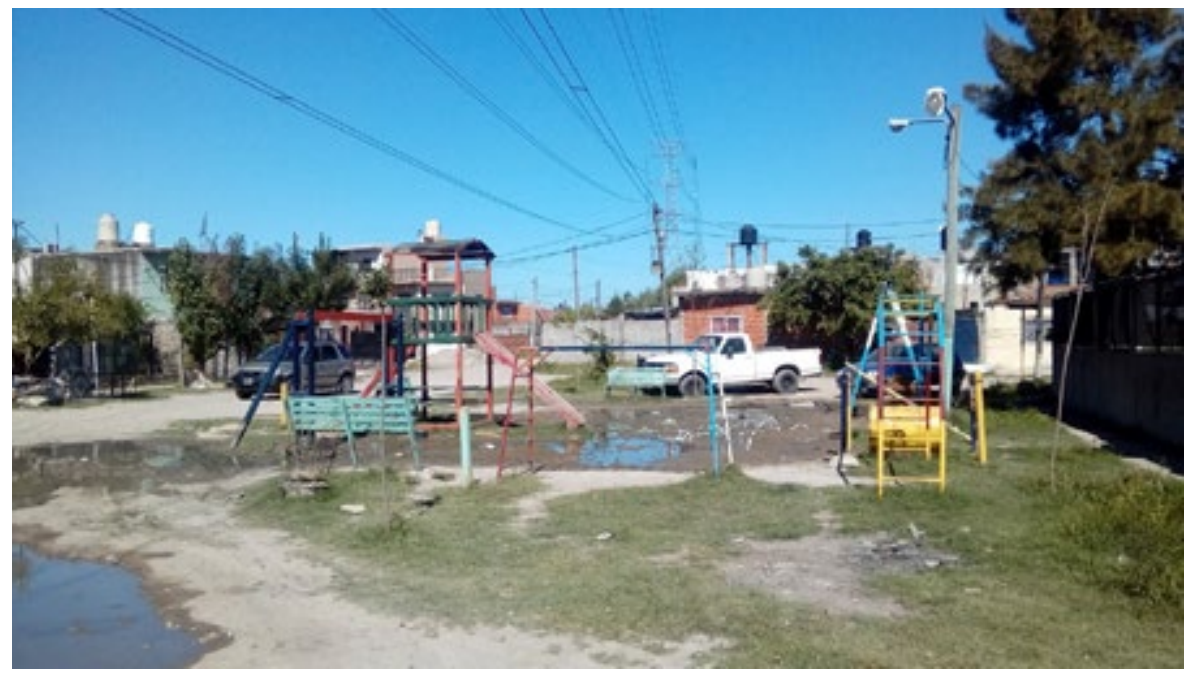

Figura 2. Veredas y espacios públicos del conurbano bonaerense, tras la lluvia. Fuente: foto de los autores, 18/03/2019.

El ordenamiento de las veredas existentes ha sido objeto de llamados de atención en distintos países, en ciertos casos inclusive con códigos propios. La proyección de espacio para la movilidad activa hacia la calzada es una medida de puesta en marcha rápida y flexible, con herramientas a mano de cualquier administración local (conos, pintura para demarcación horizontal u otras). En ciudades latinoamericanas como Buenos Aires o en ciudades europeas como las italianas, por ejemplo, se combinan con un espacio de proyección a bares y restaurants gravemente golpeados en lo económico por el aislamiento y también con necesidad de más espacio para mantener las distancias (International Transportation Forum, 2020). En otras partes, como en Barcelona, se han ampliado las veredas sobre las calzadas como proyección de un segundo patio, exterior, de las escuelas ${ }^{1}$. En el mismo sentido se inscriben las estrategias con más y mejores vías para bicicletas, no solamente en las calles y avenidas de los centros ni para viajes cortos, sino también en vías rápidas de acceso reconvertidas, pensando en trayectos de 7 o más kilómetros, tendencia que ya se venía manifestando previamente (Greater London Authority, 2018).

La reducción de velocidades máximas en las ciudades a $50 \mathrm{~km} / \mathrm{h}, 30$ o menos según los casos, mejora la convivencia entre modos de desplazamiento (World Heath Organization, 2017). Algunas ciudades, como Santa Fe en la Argentina, han adoptado por unanimidad del Concejo Deliberante una norma en ese sentido en 2020 (Figura 3). 


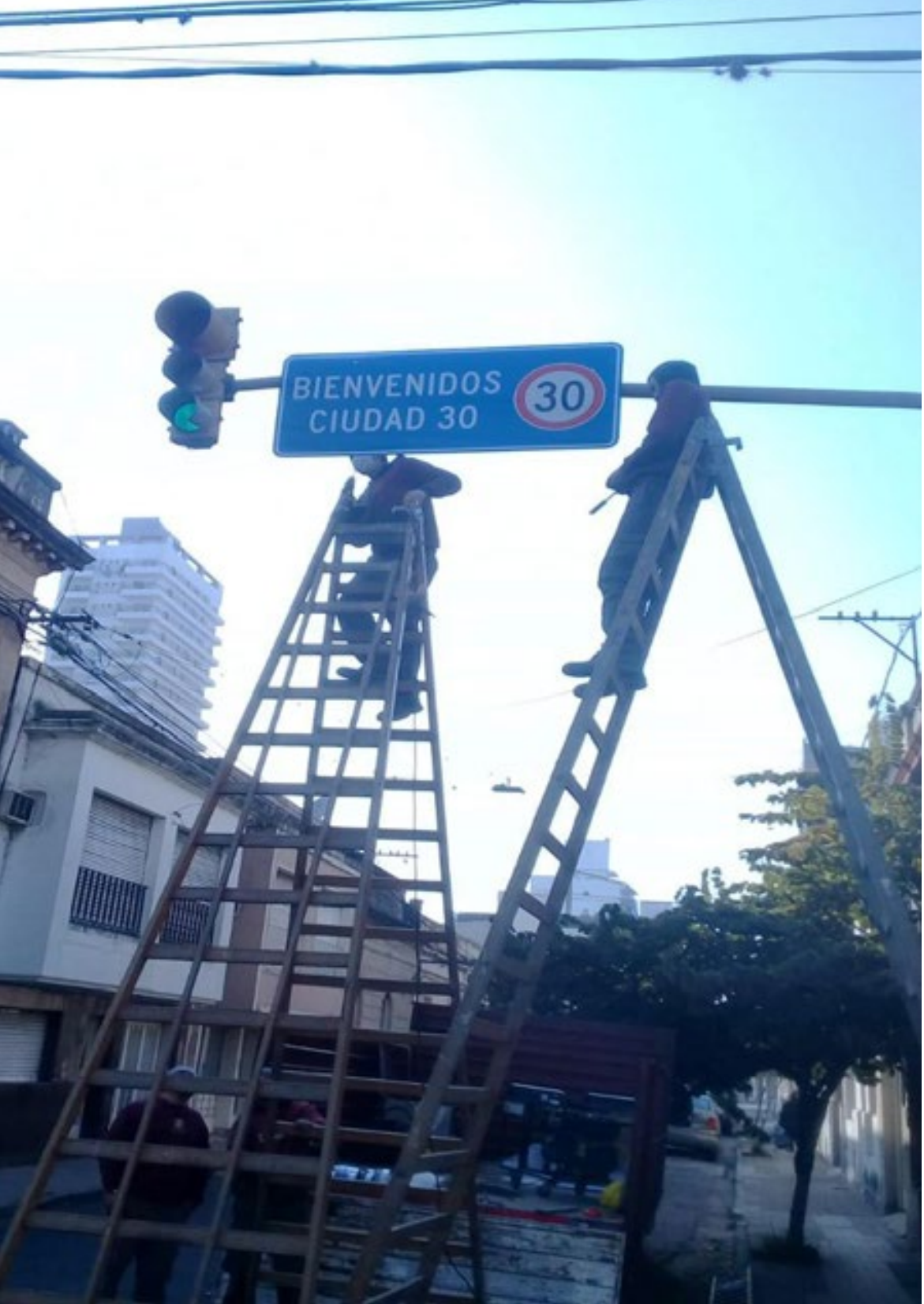

Figura 3. La instalación de carteles de acuerdo a la norma de $30 \mathrm{~km}$ por hora de velocidad máxima en Santa Fe. Fuente: gobierno municipal de Santa Fe, 2020.

Aceptar esta medida en el ámbito urbano significa, además, una reducción de costos en infraestructura, tanto en términos económicos como de transacción en el uso cotidiano que hasta hoy se venían tolerando con mayor frecuencia en ciudades latinoamericanas que en otras partes del mundo. Ejemplo de esto es la demolición de autopistas como el West Side Highway de Nueva York, las propuestas para la reconversión de vías rápidas en París, Barcelona, Amberes y Bruselas, o los cambios emblemáticos en Seúl (Lecroart, 2015).

Es cierto que existe un proceso de revisión de la forma en que la infraestructura de circulación rápida genera consecuencias negativas en las ciudades y cómo discrimina por barrios 
y niveles socioeconómicos (Dextre, 2013; Landon, 2013). Y que además existe también un movimiento reivindicativo sobre la seguridad para la movilidad activa, desde las asociaciones de ciclistas hasta ejemplos como la Liga Peatonal en México (Liga Peatonal, 2021).

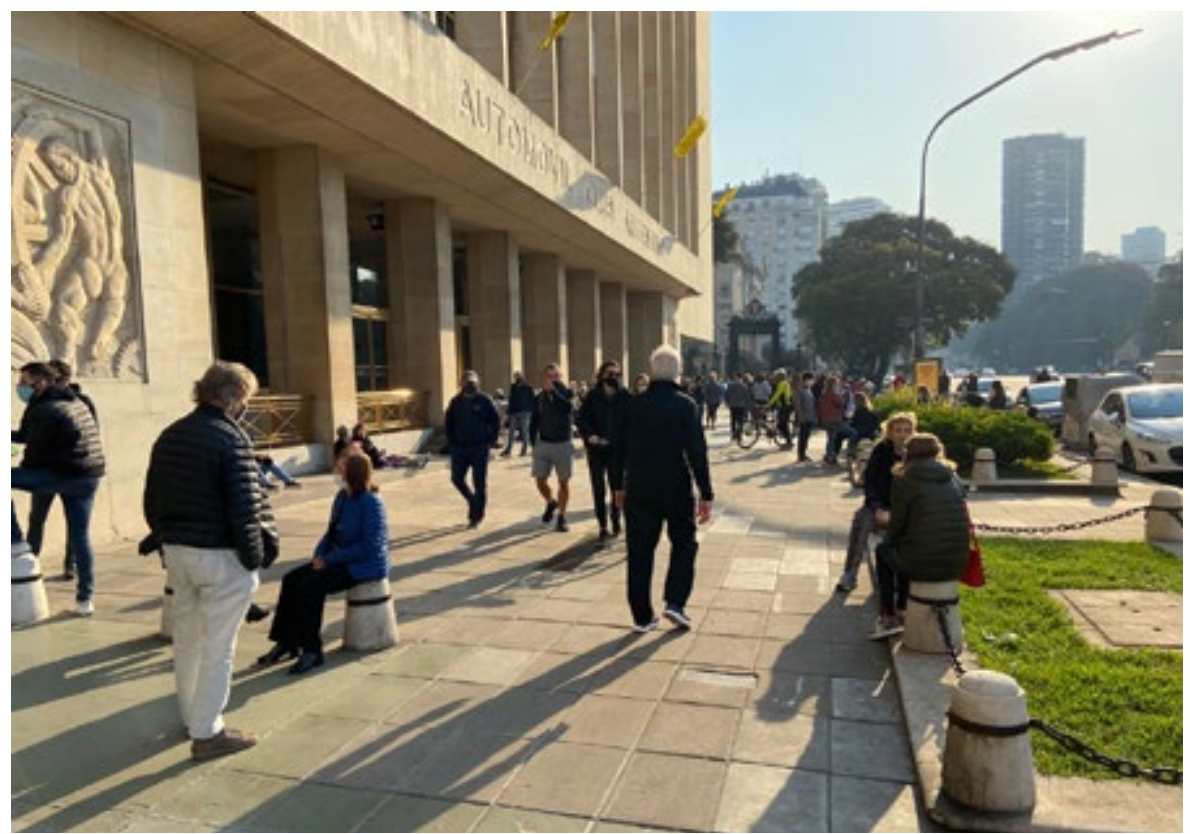

Figura 4. Peatones frente al Automóvil Club Argentino. Fuente: foto de los autores, 14/08/2020.

Pero esa concepción deberá asimilarse, multiplicarse y traducirse en acciones más contundentes y coherentes en la salida de la pandemia. En este sentido, un caso de estudio interesante para la idiosincrasia local es el acceso peatonal y en bicicleta a la Ciudad Universitaria en Buenos Aires, que depende de cruces insuficientes y corre el riesgo de verse más limitado aun, a pesar de haber sido estudiado sucesivas veces e incluso sido objeto de intervenciones públicas recientes (Borthagaray (curador), 2017; Kozak, Vecslir, Ortiz, Massin y Kuchen, 2020) (Figura 5).

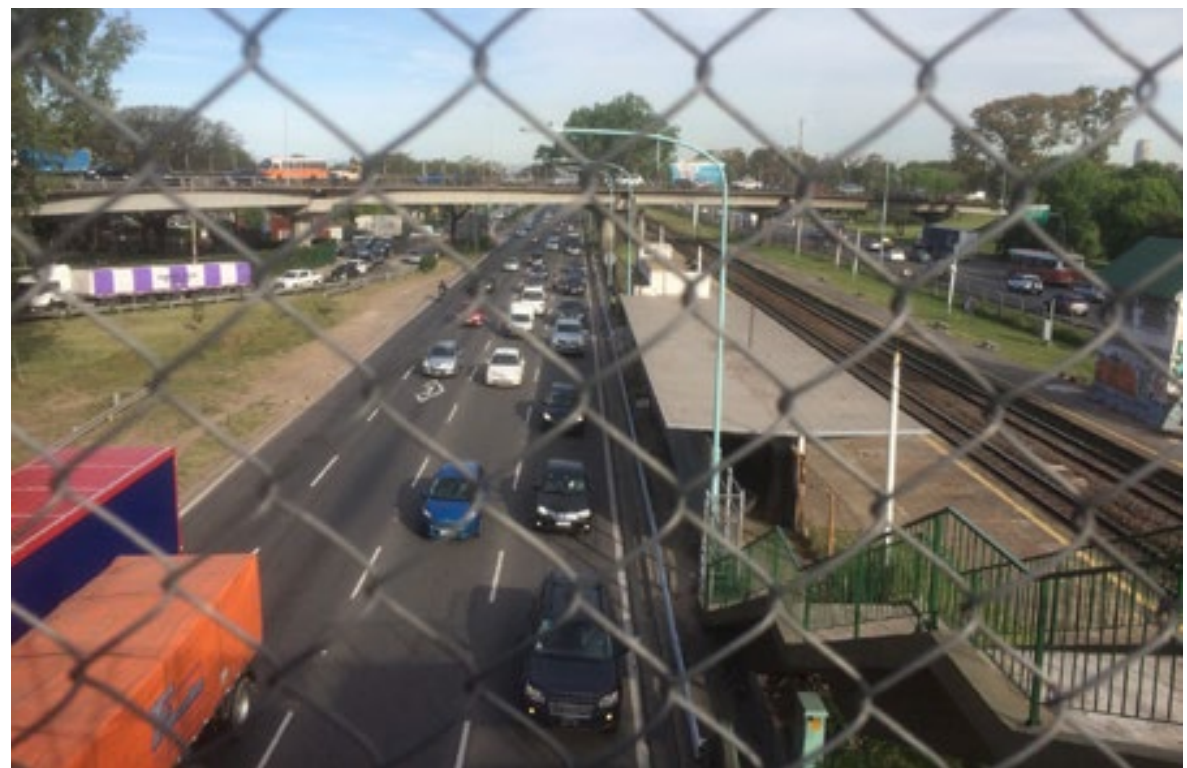

Figura 5. El acceso por puente de peatones y ciclistas a la Ciudad Universitaria. Fuente: foto de los autores, 25/10/2017. 
Está claro que, aun cuando se extremaran las condiciones que permitan un ambiente favorable para la movilidad activa, de todos modos será necesario fortalecer la oferta de un transporte público que ajuste sus servicios a una menor ocupación de las unidades y a una demanda en evolución. Con respecto al primer punto y en relación al transporte de superficie, una reducción de la capacidad a dos tercios de los espacios sentados se puede compensar en parte con prioridades claras en los recorridos, coordinación semafórica y gestión de flota (UFP-PPEC, 2020). En los subterráneos y ferrocarriles, con una frecuencia y regularidad lo mejor adaptadas e informadas que sea posible y con una mejora de las estaciones y centros de transbordo. En ambos casos extremando medidas de higiene en las superficies, las condiciones de ventilación y la protección de usuarios y trabajadores que prestan el servicio, grupo éste último particularmente expuesto (International Labour Organization, 2020a), y que no necesariamente ha tenido prioridad en las campañas de vacunación en distintos países. Finalmente, medidas como la promoción del car-pooling dentro de las ciudades con un pasajero adicional por vehículo -como lo señala José Viegas, ex responsable del ITF OCDE-, podrían tener un aumento considerable en la oferta y el uso racional del espacio vial.

El segundo punto conduce a un diálogo más profundo, entre el diseño de la oferta y la gestión de la demanda, forma de intervención llamada a tener un rol cada vez más importante a futuro, especialmente teniendo en cuenta nuevas modalidades de organización del trabajo (International Labour Organization, 2020b).

En países diversos de América Latina como Argentina, Brasil, Venezuela, Costa Rica, la cantidad de pasajeros del transporte público durante el primer semestre del 2020 rondó un zócalo entorno al 30\% del volumen habitual, respondiendo básicamente a una movilidad por trabajo. Las medidas de aislamiento o distanciamiento social y las consecuentes restricciones a la circulación tomadas en plena coyuntura, pusieron atención particular en la emergencia económica y crisis de las empresas de autotransporte público. La situación financiera de los servicios de autotransporte público del área metropolitana de Rosario -ilustrativa de las áreas metropolitanas argentinas- trajo un debate sobre las alternativas de gestión, tema de coyuntura reflejado en la prensa local (Maggi, 2021). Otros análisis del Observatorio Urbano de Córdoba recuperan un círculo vicioso de caída de ingresos cíclicos, caída de actividad y caída de pasajeros del sistema (Grupo de Investigación en Políticas de Transporte y Movilidad, 2020).

Frente a las restricciones de ocupación, el debate acerca del "nuevo normal" (Zhang, 2020) del transporte público puso foco en la supervivencia económica de la empresas prestadoras, pero también en la capacidad de financiamiento del erario público, las fuentes y modalidades de financiamiento del servicio, y finalmente, en la revisión de sus costos y fórmulas de cálculo, aspectos económicos todos estos, que no venían ocupando un lugar en la agenda latinoamericana (ni académica ni pública) acorde a su grado de necesidad. Esto llevó la atención, consecuentemente, sobre aspectos institucionales y regulatorios, y sobre aspectos territoriales, más estructurales (UFP-PPEC, 2020).

Desde el punto de vista territorial, la inadecuación entre la expansión y dinámica urbana y el diseño de las redes y servicios de transportes es un tema precedente a la pandemia, y en torno al que ya versaba la atención sobre las ciudades sustentables, bajo iniciativas presentadas como estrategia de Smart Cities o la Movilidad como Servicio (MaaS, por sus singlas en inglés) y el mercado asociado a las nuevas tecnologías (Benítez, 2019, entre otros). En las ciudades latinoamericanas, y con particular referencia al autotransporte público, coexisten problemas históricos de cobertura así como ineficiencias 
e ineficacias en el diseño de las redes existentes, tanto desde el punto de vista de los recorridos como de la gestión de flota. Esto fue algo notable en Brasil, donde en ciudades como Belo Horizonte se lograron desplegar alternativas que aumentaron la oferta con la flota existente mejorando la eficiencia y bajando costos (UFP-PPEC, 2020).

Para el caso del Área Metropolitana de Buenos Aires (AMBA), el cotejo entre la distribución territorial de la oferta y de la demanda del autotransporte público evidencia una inadecuación notoria. Mientras el 66\% de las etapas de viajes son locales (esto es, dentro de la Ciudad Autónoma de Buenos Aires -CABA- o dentro de cada municipio del aglomerado), la oferta de asientos de las líneas locales es el 20\% del total. A la inversa, las etapas de viaje entre jurisdicciones (esto es, viajes con la CABA o entre municipios del aglomerado) son el $34 \%$ del total, y se atienden con el $71 \%$ de los asientos ofrecidos (líneas provinciales y nacionales (Gutiérrez, 2020a). Según la Encuesta de movilidad Domiciliaria 2010, el 56\% de los viajes por trabajo y el $85 \%$ de los viajes por estudio, son locales. Englobando la totalidad de los motivos de viaje del AMBA, el $72 \%$ son viajes locales. Esto indica la utilidad y conveniencia de trabajar con diagnósticos espacialmente desagregados a fin de ponderar capacidades ociosas, factibles de ser cruzados con otras informaciones hoy disponibles, y contemplando motivos y necesidades específicas de viajes, como género y edad, entre otras.

Estudios comparativos de la movilidad urbana pre y post pandemia realizados con fuentes de datos tradicionales y no tradicionales para el AMBA, exponen los riesgos de una derivación hacia los viajes motorizados individuales en detrimento de los realizados en transporte público y mediante modos de movilidad activa (Anapolsky, 2020). Aplica a la Argentina y a otros muchos países del mundo el considerar entre los transportes públicos a servicios a la demanda como los taxímetros y los sistemas de bicicletas de uso compartido (en tanto servicios públicos y conforme a su capacidad específica -no masiva-).

A este último respecto es pertinente mencionar que aunque los sistemas públicos de bicicletas se expandieron por el mundo en este siglo XXI previamente a la pandemia, la cobertura territorial de aquellos que implementaron estaciones fijas sigue mayormente restringida a las ciudades centrales -e incluso a las áreas centrales- de los aglomerados urbanos. Si bien la promoción a la movilidad activa es parte de los consensos de la agenda internacional mencionados al principio, aun es difícil encontrar información oficial para evaluar cambios en los patrones de movilidad mediante una comparación con la situación previa a la pandemia, especialmente para América Latina.

La ubicuidad de los sistemas de bicicletas de alquiler sin estaciones implementados en ciudades chinas y otras partes del mundo -basados en el uso del geoposicionamiento y de aplicaciones de telefonía móvil, también para el pago ${ }^{3}$ - pone de relieve la importancia de problemas como la inseguridad urbana, que trascienden a los medios de transporte y a las posibilidades ofrecidas por las nuevas tecnologías. Sin el fundamento de estadísticas oficiales y comparables para evaluar cambios en el uso de taxis o autos de alquiler antes y a partir de la pandemia, alguna evidencia indica el refuerzo en las ciudades argentinas de un perfil de uso previo que vincula con la inseguridad urbana y con movilidades vulnerables (mujeres, infantes, ancianos). Por ejemplo, mensajes implícitos en la publicidad de empresas de las aplicaciones móviles para autos de alquiler, o en los taxis "rosa" (conducidos por mujeres) ${ }^{4}$. En este mismo sentido, si bien el

3 Videos sobre el sistema de bicicletas de alquiler en China, pueden verse en el Canal de Youtube de Andrés Pizarro https://www.youtube.com/watch?v=zDloZzJqD24 
sistema de bicicletas público más grande del país -el de la CABA- registra en 2020 el record histórico de uso (llegando a los 121 millones de recorridos), éste sigue siendo protagonizado por varones (73\%), y resultando apenas significativo el incremento de las mujeres a lo largo de una década de funcionamiento del sistema (pasando de un 23 a un $27 \%)($ GCBA, 2021) 5 .

Según el Observatorio de Movilidad y Seguridad Vial de la CABA (2021), el uso del sistema público de bicicletas en la CABA aumento de 3000 viajes en 2010 a más de 6 millones en 2019, cayendo en 2020 a valores similares al 2018 (entorno a los 2,5 millones) debido a la suspensión inicial del sistema (por dos meses) y al posterior cierre de la mitad de las estaciones (vigente hasta hoy) dispuestos como parte de las medidas a partir de la pandemia. En tanto, el número total de viajes en bicicleta (de uso público y privado) pasa de 52 millones en 2013 a 121 millones en 2020 (GCBA, 2021). Según información periodística, la venta de bicicletas durante la pandemia superó el promedio anual, llegando Argentina a tener un parque que ronda los 8,5 millones (representando alrededor del $20 \%$ de la población). Y según datos recopilados por Google Maps, Argentina se ubica en el puesto 15 en el mundo y en el puesto 3 en América Latina -detrás de Brasil y México- en relación a las consultas sobre rutas para cubrir en bicicleta (Télam Digital, 2021).

Además de apuntar a transportes públicos más competitivos y mejor adaptados a una demanda en evolución, una cuidada redistribución territorial de la oferta existente emerge como una oportunidad frente a la coyuntura de la pandemia pero también frente a estos déficits de cobertura e ineficacias de larga data. La redistribución temporal de la oferta y su regularidad, la coordinación horaria con actividades esenciales (como escuelas, hospitales) es un auxilio al folklórico pendular entre colectivos abarrotados y largas filas de espera en algunos horarios, y unidades vacías en otros. Por otro lado, la existencia de prioridades del transporte público en la circulación alcanza todavía pocos kilómetros, con notorias ausencias en los principales puntos de acceso vial dentro del AMBA.

Una organización diferente de horarios, espacios y prácticas de trabajo, podrá potenciarse con planes de movilidad por empresa. Claramente, el teletrabajo es la práctica más descollante del escenario de la pandemia (Giniger, 2020). A pesar de que este escenario aún no ha finalizado, se registran vaivenes en las opiniones acerca de su perdurabilidad, desde un progresivo consenso inicial acerca de su consumación hasta algunas dudas más recientes según se recuperan pros y contras para los trabajadores y las empresas, tras la experiencia de los últimos meses (Bolotsky, 2020). Cuestiones como la regulación laboral del teletrabajo ${ }^{6}$, las condiciones de trabajo, el tiempo y la organización del trabajo, el equilibrio entre la vida laboral y la privada (permeado por cuestiones de género) (CEPAL, 2020), el desempeño laboral, la supervisión y vigilancia de los empleados, la cultura organizativa empresarial y las economías de costo, están bajo la observación y sin un cierres conclusivos. Desde la gestión de la demanda, una organización diferente de horarios, espacios y prácticas de trabajo, podrá potenciarse con planes de movilidad por empresa u organización.

que la comodidad. Con respecto a los taxis "rosa" puede verse, por ejemplo https://www.ellitoral.com/index.php/ id_um/274761-una-sanjuanina-ofrece-un-servicio-de-taxi-rosa-solo-para-mujeres-ancianos-o-ninos-de-confianzacambalache.html

5 Sobre la expansión del sistema público de bicicletas en la CABA puede consultarse https://poblaciones. org/2021/04/o8/estaciones-ecobici-y-red-de-ciclo-vias-2010-2019 
Desde un punto de vista más estructural, se evidencia la necesidad de revisar las categorías ocupacionales mediante las cuales se relevan y producen datos. Para administrar la movilidad por trabajo es necesario conocer las prácticas territoriales y generar información según el grado de dependencia o independencia espacial con respecto al lugar de trabajo y/o las instalaciones del empleador (Gutiérrez, 2020b) con una profundidad que las actuales fuentes estadísticas oficiales no permiten recabar o desagregar a nivel intra metropolitano y local (Batty, 2020). Cabe destacar que esto se inscribe hoy en un contexto donde la "oficina" tradicional se plantea en extinción, o por lo menos en un cambio de uso significativo (Blasco, 2020).

En el mismo sentido, la actividad educativa presencial podrá alternar con la actividad virtual, y en niveles superiores incluso ampliar la cobertura geográfica a escala nacional e internacional de los estudios para ciertas especializaciones. Por supuesto, en los niveles obligatorios y en situaciones de mayor vulnerabilidad, eso requerirá redoblar los esfuerzos de inclusión por parte de los gobiernos, atendiendo tanto la adaptación de la infraestructura edilicia y de los servicios como de los recursos humanos y pedagógicos, de los procesos administrativos y de gestión involucrados, todos mediados por una ingeniería de compleja de consensos y articulaciones.

En general, las finalidades de muchos desplazamientos, especialmente por acceso a servicios, trámites y cuestiones administrativas, pudieron resolverse progresivamente de manera on line en lo que va de la pandemia, y seguramente podrán continuar haciéndolo. En cuanto a los comercios, sus horarios podrán ofrecer una organización escalonada, aunque es destacable aquí el crecimiento exponencial del comercio electrónico, que merma los desplazamientos por parte de los consumidores pero induce otros asociados a la logística y distribución final de los bienes, lo que pone a prueba el rol regulador de las autoridades, incluyendo riesgos de seguridad vial y precariedad laboral, además de acciones de gestión y planeamiento, vinculadas con el ordenamiento del espacio público y transformaciones territoriales con impacto en el mercado inmobiliario, entre otras (Gutiérrez, Ziliani, y Borthagaray, 2019).

Esta combinación de experiencias y posibilidades se expresan en el territorio y con un impacto diferencial. Esto permite proyectar algunos escenarios, particularmente en las avenidas comerciales y en las áreas centrales de las regiones metropolitanas. Por una parte, si bien el cierre de comercios de avenida puede recuperarse en parte con el levantamiento pleno de las restricciones, el crecimiento del comercio electrónico tiende a seguir aumentando proporcionalmente de todos modos (Figura 5). 


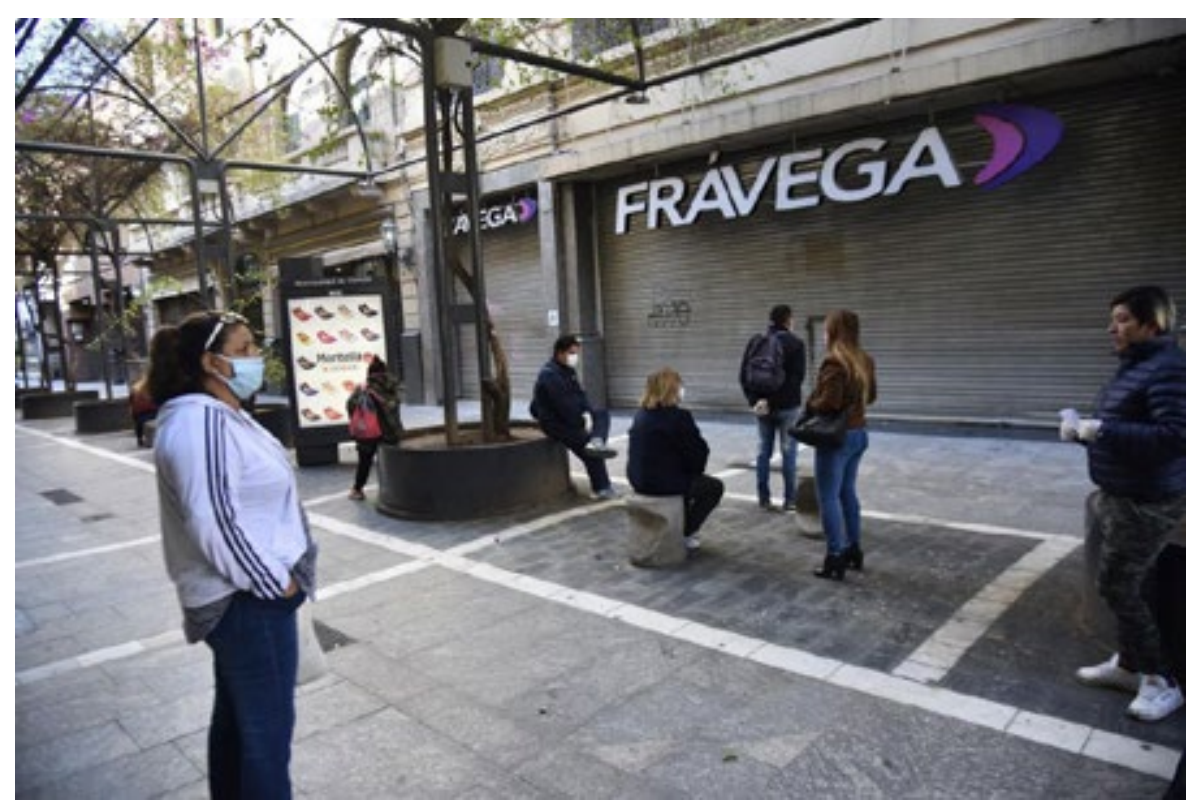

Figura 5. Una imagen típica de áreas centrales de ciudades argentinas durante las restricciones sanitarias más severas. Fuente: Pedro Castillo, La Voz del Interior, Córdoba, 2020.

Por otra parte, la concentración de actividades y servicios especializados en áreas centrales padece una situación coyuntural de crisis, compartida por el microcentro porteño con otros grandes centros urbanos, y que se refleja también en el sector inmobiliario (Chen, 2020; Ocvirk, 2021).

\section{¿Movilidad urbana post pandemia?: Fuerzas en pugna}

Las tendencias mencionadas, más generales, se asimilan y traducen en el ámbito local conforme a factores demográficos y urbanísticos, políticos e institucionales (desde el punto de vista de las acciones y normativas públicas) entre otros que pueden ilustrarse a través del caso de la Ciudad Autónoma Buenos Aires: la pérdida de población en un proceso de largo plazo, los incentivos fiscales para la radicación de actividades en otros distritos de la ciudad, el desarrollo de polos como el de Av. Gral Paz y Panamericana (Usatinsky, 2018), la multiplicación de ofertas competitivas en lugares menos servidos por transporte público y la mudanza de oficinas gubernamentales que ha multiplicado la pérdida de actividad, como lo ilustra desde hace algún tiempo la situación del ex Mercado del Plata (Frittaoni, 2018). Se suman a estos factores un abanico de intervenciones en transporte, que conviven en un mismo y significativo recorte territorial como es el macrocentro porteño, y cuyas apuestas pugnan por la construcción de infraestructuras orientadas al uso del automóvil y del transporte público. Tales la multiplicación de accesos y salidas de la autopista Illia (Legislatura de la Ciudad de Buenos Aires, 2009) y la construcción del llamado Paseo del Bajo (Gobierno de la Ciudad de Buenos Aires, 2019), por un lado; y la prolongación de la línea E de subterráneos (de la estación Bolívar a Retiro) y las prioridades a la circulación de colectivos por el llamado Metrobus (en las Avenida 9 de Julio y Leandro Alem-Paseo Colón al norte de la Avenida Brasil), por el otro. Podría ser que estas apuestas no pugnaran en distintos sentidos sino que buscarán un balance en los efectos inducidos (ambientales, sociales y territoriales), sin embargo, aquellas en pos del transporte público han tenido un derrotero diferencial: la línea E no llegó a desarrollar una frecuencia atractiva y el Metrobus todavía necesita de aliviadores en la oferta norte sur, como la postergada línea F de Subterráneos 
y el desarrollo final de la línea H (Mardjetko, 2021). Finalmente, ambas apuestas se combinan con una política de prioridad a los peatones, -inscripta en las tendencias internacionales. Así, a la vez que la orientación al automóvil induce, entre otros efectos, a la contaminación y los accidentes, el Metrobus mejora problemas ambientales pero con efectos colaterales, como el desplazamiento de los colectivos que circulaban por el par Esmeralda-Maipú a la Avenida 9 de Julio, dejando sin oferta de transporte público una distancia de 8 o más cuadras según los tramos, entre la Avenida 9 de Julio y las avenidas del "bajo"7.

La movilidad post pandemia significa más que una reserva peatonal circunscripta a un conjunto de calles o distritos. Significa, sobre todo, una ciudad caminable globalmente con una política articulada en todos los sentidos, orientada a evitar problemas de consistencia (explícita o implícita).

Tomando en cuenta lo complejo del punto de partida y lo incierto del futuro, es difícil aventurarse a sostener proyecciones. Aunque hay al menos dos certidumbres que pueden sostenerse acerca de la evolución de la pandemia. Una, que sea ésta cual fuere, la vulnerabilidad (socioeconómica y territorial) no desaparecerá. Y dos, que ninguna proyección será lineal, sino bien nutrida de contingencias e incertidumbres, conflictos y contradicciones.

Pero la pandemia sí habrá ampliado las compuertas para el paso de formas innovadoras (preexistentes o emergentes) en la organización social de los desplazamientos en las ciudades, y alumbrado la posibilidad de afrontarlas sobre la base de la movilización extraordinaria de voluntades políticas y conductas sociales que generó (Florida y Kotkin, 2021). Cambios necesarios para hacer frente a una situación que ya era insostenible antes de la pandemia, por la crisis del calentamiento global, la desigualdad y el endeudamiento. Una crisis frente a la que no alcanzaba con un mero cambio en la fuente de energía de los vehículos, de un sistema de combustión interno a uno eléctrico, ni con una ilusión tecnocrática sobre la automatización y las tecnologías de la información. Por más que existan innovaciones positivas en uno y otro sentido, sumadas al aporte de la conectividad y de una disponibilidad inédita de datos en tiempo real, aún restan pasos impostergables en la organización social, territorial e institucional de la movilidad, entre ellos, por ejemplo, la generación de información de calidad y el acceso a misma (Benítez, 2019).

$\mathrm{Y}$ en estos pasos median componentes materiales e inmateriales, pasados y presentes. Recuperando a Milton Santos y su metáfora sobre la "rugosidad" del territorio (Santos, 1986) como una inercia dinámica del pasado en el presente, una inercia morfológica de naturaleza social, que resiste o retarda las nuevas realidades, ésta será una fuerza actuante en el devenir de la post pandemia. Pero como amplía Mónica Arroyo (1996), dicha inercia territorial no es sólo una morfológica, sino una combinación de materialidades (naturales o fabricadas, técnicas o informacionales) y de acciones (económicas, políticas, culturales, premeditadas, automáticas o espontáneas, hegemónicas o contra hegemónicas, plásticas o rígidas). Así, las morfologías son constituidas histórica, dinámica y permanente, y pueden ser re significadas por nuevos contenidos, siendo o no sustituidas, sea total o parcialmente (Arroyo, 1996).

Frente a la inercia del territorio y de los hábitos, la emergencia generó un quiebre que aceleró y habilitó cambios marcados. Habrá, sin dudas, una tendencia a restaurar la "homeostasis" anterior. Pero también habrá nuevas fuerzas en pugna, emergentes de la 
situación. Esta encrucijada aún coloca una oportunidad histórica y única, que puede mal gastarse o capitalizarse para hacer más real las aspiraciones internacionales que, con llamativo consenso, organizaban la agenda urbana pre pandemia. Una oportunidad marcada por la posibilidad de adecuar la oferta de transporte a un nuevo tipo de demanda de movilidad, de hacer más competitiva una movilidad más sustentable, más inteligente en el uso de los recursos, más inclusiva socialmente y más equitativa territorialmente.

El devenir de un proyecto de ciudad y de movilidad seguirá requiriendo de arbitrajes políticos de demandas en conflicto, disputas y criterios para la asignación de recursos públicos, una cultura para organizar la articulación tanto vertical como horizontal entre las instituciones públicas y con la investigación científica aplicada, y de una ética que aspire a llevar la participación ciudadana más allá de lo formal, y de manera plural.

Y la realidad apremia. Aunque muchas de las experiencias acá hilvanadas son fundamentadas en casos o estudios parciales de algo que todavía está en desarrollo, las medidas de recuperación post pandemia ya están siendo sopesadas o implementadas buscando alternativas inmediatas de asignación de recursos (millonarios), basadas en la reflexión previa disponible. Tales los casos del Recovery Fund europeo o del plan de infraestructura en debate, propuesto en Estados Unidos a partir -entre otros elementosde una progresiva reapreciación de las consecuencias en el territorio de intervenciones disruptivas en la infraestructura de transporte. Un relevamiento de las situaciones previas y posteriores a la construcción de autopistas en barrios consolidados muestra efectos de segregación social a partir de la ruptura del tejido urbano. Es cierto que son montos de inversión importantes (Popovich, Williams, y Lu, 2021). Pero en todo caso, existen mayores fundamentos para evitar asignar recursos públicos a intervenciones que generan efectos negativos difíciles de revertir en primer lugar.

Esperamos que este ensayo logre hilvanar un panorama de experiencias somero pero capaz de despuntar la exploración posterior de esta encrucijada histórica, indispensable de ser indagada en profundidad y a largo plazo, pero también de ser caminada mediante el sondeo de hipótesis aun cuando no estén todos los elementos disponibles.

Aclaraciones: Este artículo se basa en parte en reflexiones previas, individuales o compartidas, como por ejemplo: "Contribución para 100 reflexiones en tiempos de pandemia", "Hiperlugares móviles, actividades conectadas más allá del transporte", "Espacio de la movilidad post fósil" o el seminario virtual "Desafíos territoriales frente a la pandemia del COVID-19", organizado por el Instituto de Geografía de la UBA. 


\section{O Bibliografía}

» Anapolsky, S. (2020). ¿Cómo nos movemos en el AMBA? Conclusiones de la evidencia empírica y alternativas post-Covid. Serie: Documentos de Trabajo del IT - UNSAM, 18 (Septiembre), 1-21.

» Angel, S., Blei, A. M., Lamson-hall, P., y Salazar, M. M. (2020). The Coronavirus and the cities: explaining variations in the onset of infection and in the number of reported cases and deaths in U.S. metropolitan areas as of 27 March. New York University https://marroninstitute.nyu.edu/uploads/content/ The_Coronavirus_and_the_Cities\%2C_27_March_data\%2C_final_draft_31_ March_2020_VersApril3.pdf

"Anzillotti, A. (2021). El pulso de la pandemia sacudió el mercado en los suburbios. Retrieved from https://noticias.argenprop.com/actualidad/elpulso-de-la-pandemia-sacudio-el-mercado-en-los-suburbios/ (20/05/2021).

"Arroyo, M. (1996). A trama de um pensamento complexo: espaco banal, lugar e cotidiano. En C. A. Fanni Alessandri (Ed.), Ensaios de geografia contemporãnea. Milton Santos obra revisitada (pp. 52-62). Sao Paulo: Hucitec.

»Batty, M. (2020). The coronavirus crisis : what will the post-pandemic city. Environment and Planning B: Urban Analytics and City Science https://doi. org/10.1177/2399808320926912

»Benítez, C. (editora). (2019). Cómo aplicar big data en la planificación del transporte urbano. El uso de datos de telefonía móvil en el análisis de la movilidad. Retrieved from https://publications.iadb.org/publications/spanish/ document/Como_aplicar_big_data_en_la_planificación_del_transporte_ urbano_El_uso_de_datos_de_telefonía_móvil_en_el_análisis_de_la_ movilidad_es.pdf

"Blasco, L. (2020). Coronavirus y teletrabajo: 5 modelos de "oficina del futuro" que están emergiendo gracias a la pandemia. Bbc.Com. Retrieved from https:// www.bbc.com/mundo/noticias-54356853 (04/10/2020).

»Bolotsky, M. (2020). ¿Ha llegado el teletrabajo para quedarse? Cómo el coronavirus puede cambiar el futuro del trabajo. Retrieved from https://news. un.org/es/story/2020/06/1475242 (20/05/2021).

»Borthagaray (curador), A. (2017). La mutación del espacio urbano en la era post fósil. Arquis, Revista de Arquitectura de la Universidad de Palermo, 1 (8), 55. Retrieved from https://www.palermo.edu/arquitectura/pdf/Arquis_ POSTFOSIL.pdf

»Comisión Económica para América Latina y el Caribe (CEPAL) (2020). Cuidados y mujeres en tiempos de COVID-19: la experiencia en la Argentina. Documentos de Proyectos (LC/TS.2020/153). Santiago: CEPAL.

"Chen, S. (2020). The Real Estate collapse of 2020. The pandemic devastated the housing industry this year, but there were a few flickers of life. The New York Times. Retrieved from https://www.nytimes.com/2020/12/25/realestate/nycreal-estate-market.html (21/12/2020).

»Deaton, A. (2015). El Gran Escape. Salud, riqueza y los orígenes de la desigualdad. Buenos Aires: Fondo de Cultura Económica.

»Dextre, J. C. (2013). Los peatones son los más vulnerables de la ciudad: una 
mirada desde el punto de vista de la equidad. En J. P. Orfeuil \& A. Borthagaray (Eds.), La Fábrica del Movimiento. 16 casos de política pública en materia de movilidad urbana. (pp. 33-38). Buenos Aires: Café de las Ciudades.

»Fernández, S., Scholl, L. y IADB. (2020). Asentamientos informales y movilidad. Retrieved from https://transporteterritorio.wixsite.com/pttuba/audiovisual

" Florida, R. y Kotkin, J. (2021). America's Post-Pandemic Geography Covid-19 is transforming all types of communities, from big cities to suburbs to rural areas. City Journal. (Spring).

» Frittaoni, V. (2018). Vacío hace 2 años, empeora el entorno del Edificio del Plata y los comerciantes dicen que pierden clientes. Clarín. (06/04/2018).

» Giniger, N. (2020). Teletrabajo en tiempos de pandemia. Retrieved from http:// www.ceil-conicet.gov.ar/2020/o5/teletrabajo-en-tiempos-de-pandemia-nuriaginiger/ (28/05/2021).

» Gobierno de la Ciudad de Buenos Aires. (2021) Análisis del uso de la bicicleta en la Ciudad Autónoma de Buenos Aires. Año 2010-2020. Observatorio de Movilidad y Seguridad Vial de la Ciudad de Buenos Aires.

» Gobierno de la Ciudad de Buenos Aires. (2019). Construir el Paseo del Bajo. Retrieved from https://www.buenosaires.gob.ar/compromisos/paseo-del-bajoconectar-illia-au-la-plata (29/04/2021).

» Greater London Authority. (2018). Mayor's Transport Strategy. https://www. london.gov.uk/sites/default/files/mayors-transport-strategy-2018.pdf

» Grupo de Investigación en Políticas de Transporte y Movilidad (GIPTM). (2020). Reflexión sobre la situación de la movilidad en la ciudad de córdoba en el marco de la pandemia producida por el covid-19 Retrieved from https://ouc. unc.edu.ar/investigaciones/ (30/05/2021).

"Gutiérrez, A. (2012). Movilidad y territorio. Herramientas para la integración sectorial del ordenamiento a escala barrial. II Seminario Internacional Procesos Urbanos Informales. Bogotá: Universidad Nacional de Colombia.

»Gutiérrez, A. (2020). En el corazón de la pandemia...laten el transporte y el territorio. Desafíos hacia una movilidad útil. Seminario virtual: Desafíos territoriales frente a la pandemia del COVID-19, IIGEO-UBA, Buenos Aires. https:// www.youtube.com/watch?v=mszBqgjQnZ8\&feature=youtu.be (19/5/2020).

"Gutiérrez, A. (2020a). A buen entendedor, pocas palabras: el futuro del autotransporte público invita a una redistribución territorial de la oferta existente (y hoy ociosa). In Medidas de rescate para el sector del transporte público en la pandemia. PPIC-UFPerbambuco, Brasil. https://www. academia.edu/43492976/A_buen_entendedor_pocas_palabras_el_futuro_ del_autotransporte_p\%C3\%BAblico_invita_a_una_redistribuci\%C3\%B3n_ territorial_de_la_oferta_existente_y_hoy_ociosa_

"Gutiérrez, A. (2020b). Movilidad por trabajo y potencial de teletrabajo en el AMBA. Exploratoria de datos y categorías de análisis con base en las Encuestas de Movilidad Domiciliaria. https://www.academia.edu/43492949/Movilidad_ por_trabajo_y_potencial_de_Teletrabajo_en_el_AMBA_Exploratoria_ de_datos_y_categor\%C3\%ADas_de_an\% C3\%A1lisis_con_base_en_las_ Encuestas_de_Movilidad_Domiciliaria

»Gutiérrez, A., Ziliani, L., y Borthagaray, A. (2019). La ville lise, flux et points d'ancrage de l'e-commerce à Buenos Aires. Urbanisme, 65-68.

»Intergovernmental Panel on Climate Change (IPCC). (2018). Summary for urban 
policy makers: what the Special Report on Global Warming of $1.5^{\circ}$ means for cities. https://doi.org/10.24943/SCPM.2018

"International Energy Agency. (2021). Net Zero by 2050 A Roadmap for the Global Energy Sector. Retrieved from https://iea.blob.core.windows. net/assets/4719e321-6d3d-41a2-bd6b-461ad2f850a8/NetZeroby2050ARoadmapfortheGlobalEnergySector.pdf

» International Labour Organization. (2020a). ILO Sectoral Brief, (May 2018), 1-10.

" International Labour Organization. (2020b). Policy Brief Working from Home: A potential measure for mitigating COVIOD-19 pandemic, (April), 1-10.

»International Transportation Forum. (2020). Re-spacing Our Cities For Resilience. COVID-19 Transport Brief. Analysis, facts and figures for transport's response to the coronavirus. Paris.

»International Transportation Forum. (2021). Decarbonising Transport in Latin American Cities. https://www.itf-oecd.org/dtla-final-event

" Kamin, D. (2021). The market tectonics of California Real Estate. The New York Times.

»Kozak, D., Vecslir, L., Ortiz, F., Massin, T. y Kuchen, E. (2020). Patrones de movilidad en Ciudad Universitaria, UBA. Una distribución modal más sustentable. IV CICSE, 1-28.

"Landon, P. (2013). Movilidad cotidiana e infraestructura vial: nuevos desafíos urbanos para la inclusión social en la ciudad. El caso de la autopista acceso a Santiago. Revista de Trabajo Social, 84(Agosto), 31-45.

»Lecroart, P. (2015). La ville après l'autoroute: renaissances urbaines? Expériences et leçons pour le Grand Paris au menu. Paris: IAURIF.

»Legislatura de la Ciudad de Buenos Aires. Ley 3060 (2009). Buenos Aires. Retrieved from http://www2.cedom.gob.ar/es/legislacion/normas/leyes/ ley306o.html

" Liga Peatonal. (2021). Puentes antipeatonales. Retrieved from https://www. ligapeatonal.org/puentes-antipeatonales/ (30/05/2021).

" Maggi, N. (2021). En medio de la crisis resurge la idea de estatizar el transporte público. La Capital. La Ciudad.

»Mardjetko, L. (2021). Análisis técnico de la línea H de Subterráneos de Buenos Aires a diez años de su inauguración. Universidad de Buenos Aires.

"McMahon, J. (2020, Marzo 16). Study: coronavirus lockdown likely saved 77,000 lives in China just by reducing pollution. Forbes. Retrieved from https://www. forbes.com/sites/jeffmcmahon/2020/03/16/coronavirus-lockdown-may-havesaved-7700o-lives-in-china-just-from-pollution-reduction/\#1do5138234fe (16/03/2020).

»Naciones Unidas (2017). Nueva Agenda Urbana. Ecuador: ONU.

"Ocvirk, V. (2021, Febrero 22). Oficinas vacías: ¿qué será del microcentro y cómo resucitarlo? La Nación. Retrieved from https://www.lanacion.com. ar/propiedades/inmuebles-comerciales/oficinas-vacias-que-sera-delmicrocentro-y-como-resucitarlo-nid22022021/ (22/02/2021).

»Popovich, Na., Williams, J., y Lu, D. (2021, Mayo 28). Can Removing Highways Fix America's Cities? The New York Times. (28/05/2021).

»Redacción de La Voz, y Foto: Pedro Castillo, La Voz. (2020). En fotos: el centro 
de Córdoba, con poco movimiento. La Voz. Retrieved from https://www.lavoz. com.ar/galerias/en-fotos-centro-de-cordoba-con-poco-movimiento

"Santos, M. (1986). Por uma geografia nova. São Paulo: Hucitec. (1ª ed., 1978). Trad. cast. (1990) Por una geografía nueva. Madrid: Espasa Calpe.

» Télam Digital (2021) Una tendencia que, en Argentina y en el mundo, anda sobre dos ruedas

" https://www.telam.com.ar/notas/202106/556379-dia-mundial-de-la-bicicletaonu-pandemia-sustentable.html (03/06/2021).

» Universidade Federal de Pernambuco - Programa de Pós-graduação em Engenharia Civil (UFP-PPEC). (2020). Forum de Discusión Medidas de rescate para el sector del transporte público en la pandemia, Lima Neto, O (org.), 1er. semestre de 2020, Brasil.

»Usatinsky, M. E. (2018). Panamericana y General Paz: una zona en ebullición. La Nación. Retrieved from https://www.lanacion.com.ar/propiedades/inmueblescomerciales/panamericana-y-general-paz-una-zona-en-ebullicion-nid2142263/ (11/06/2018).

»World Health Organization. (2009). Global Status Report on Road Safety. Time for action.

»World Heath Organization. (2017). Managing speed. Villars-sous-Yens.

"Zhang, J. (2020). Transport policymaking that accounts for COVID-19 and future public health threats: A PASS approach. Transport policy, 99, 405-418.

\section{Otras lecturas}

»Viegas, J. https://www.publico.pt/2020/05/o4/opiniao/opiniao/mobilidadeurbana-periodo-distanciamento-social-1914448

»Orfeuil, J. P https://www.ville-en-mouvement.com/fr/content/mortalite-covidet-densite-des-territoires-les-differences-de-mortalite-en-france-par

»Baruchman, M. https://www.seattletimes.com/seattle-news/transportation/ seattle-will-permanently-close-20-miles-of-residential-streets-to-mostvehicle-traffic/

"Soames Job, B. https://blogs.worldbank.org/transport/can-covid-19-teach-ussomething-road-safety-epidemic

»European Commission https://ec.europa.eu/transport/coronavirus-response_en

»CIPPEC https://www.cippec.org/textual/teletrabajo-para-una-nueva-normalidadque-podemos-aprender-de-las-empresas-que-lideran-la-innovacion-enargentina/

\section{Protocolos de seguridad}

»https://www.tc.gc.ca/en/initiatives/covid-19-measures-updates-guidance-tc. html

》 https://www.ecdc.europa.eu/sites/default/files/documents/COVID-19-publictransport-29-April-2020.pdf 


\section{Andrés Borthagaray / aborthagaray@gmail.com}

Arquitecto de la Facultad de Arquitectura, Diseño y Urbanismo de la Universidad de Buenos Aires (UBA), diploma internacional en administración pública (ENA), doctor en urbanismo y geografía, Sorbonne nouvelle. Director del posgrado en diseño y gestión de ciudades inteligentes e inclusivas (FADU UBA), entre otras actividades docentes. Presidente de la Fundación Furban y director del Instituto para la Ciudad en Movimiento, América Latina.

\section{Andrea Gutiérrez / angut2@gmail.com}

Licenciada y Doctora en Geografía de la Universidad de Buenos Aires (UBA). Investigadora CONICET y Profesora Asociada del Departamento de Geografía (UBA). Directora del Programa Transporte y Territorio, y de la Revista Transporte y Territorio (Instituto de Geografía, UBA). 